\title{
Scanning Precession Electron Diffraction to aid Aluminum Alloy Development
}

\author{
Jonas K. Sunde ${ }^{1}$, Emil Christiansen ${ }^{1}$, Elisabeth Thronsen $^{1}$, Sigurd Wenner ${ }^{1,2}$, Calin D. Marioara ${ }^{2}$, \\ Antonius T.J. van Helvoort ${ }^{1}$, Randi Holmestad ${ }^{1 *}$ \\ 1. Department of Physics, Norwegian University of Science and Technology (NTNU), N-7491 \\ Trondheim, Norway \\ 2. Materials and Nanotechnology, SINTEF Industry, N-7465 Trondheim, Norway \\ * Corresponding author: randi.holmestad@ntnu.no
}

In this work, we demonstrate how scanning precession electron diffraction (SPED) is used to study the microstructure of age-hardenable aluminum alloys. These alloys, based on Al-Mg-Si (6xxx), Al-Mg-Zn $(7 \mathrm{xxx})$ and $\mathrm{Al}-\mathrm{Cu}(2 \mathrm{xxx})$, are important structural materials for construction and automotive applications due to properties like high strength/weight ratio and good formability, often combined with good corrosion resistance. Our overall objective is to improve the understanding of the fundamental physics taking place at the different length scales in these alloys - from grains, grain boundaries and precipitation free zones, through primary particles and dispersoids, down to nanometer sized precipitates and atomic clusters. The distribution, number density, morphology, structure and interfaces of dispersoids and precipitates depend on the alloy composition and the thermo-mechanical history of the material, and determine to a large extent the material's physical properties. If we are able to quantify and predict the microstructure evolution during thermal aging or mechanical deformation, we can design new and optimize chemical compositions of existing alloys to get desired properties for a given application. We will here demonstrate how SPED enables extraction of important information from different key constituents of the $\mathrm{Al}$ alloy microstructure, providing good statistics by analyzing relatively large areas $\left(\sim 1 \mu \mathrm{m}^{2}\right)$ while maintaining spatial resolution down to a few nanometers [1-4]. Combined with high resolution (scanning) transmission electron microscopy ((S)TEM), this allows for better prediction and design of alloys and heat treatments for industrial applications.

In SPED a nm-sized, precessing beam is raster scanned across the sample, and a diffraction pattern $\left(\mathrm{k}_{\mathrm{x}}, \mathrm{k}_{\mathrm{y}}\right)$ is recorded at each probe position $(\mathrm{x}, \mathrm{y})$, forming a 4D data set. All SPED experiments were performed using a JEOL 2100F (S)TEM operating in nanobeam diffraction mode at $200 \mathrm{kV}$ using a NanoMEGAS ASTAR system. The probe convergence semi-angle was $1.0 \mathrm{mrad}$ and precession angles employed were $0-1.0^{\circ}$ with a precession frequency of $100 \mathrm{~Hz}$. The scan step size was in the range $0.76-$ $2.28 \mathrm{~nm}$ and the exposure time per pixel was 10-40 ms. Diffraction patterns were acquired using a Stingray camera recording the microscope's fluorescent screen. The double-rocking probe required for PED was aligned following the method detailed by Barnard et al. [5]. A typical data set can cover up to $1 \mu \mathrm{m}^{2}$ areas, comprising $\sim 500^{2}$ diffraction patterns $(\approx 5 \mathrm{~GB})$ and requiring $\sim 1-2$ hours acquisition time. The acquired 4D data set can readily be explored using multidimensional data processing packages, such as HyperSpy and pyXem [6,7]. Figure 1 shows how the SPED data can be visualized, using virtual dark field (VDF) imaging formed by the use of a virtual aperture positioned in the PED pattern stack. The data is visualized using a graphical user interface (GUI) built on HyperSpy. Digital post processing is done by various data analysis routines, including virtual imaging, machine learning and template matching.

SPED can be used to quantify the distribution of phases and their subsequent evolution with ageing. In combination with STEM, phases and precipitate phase fractions in different ageing conditions can be quantified through analysis of a statistically significant number of precipitates [1], as shown in Figure 2. Moreover, with a clever use of VDF imaging, SPED is also ideal to study precipitates in heavily 
deformed alloys, where the contrast from dislocations often obscure all other features in conventional imaging techniques [2]. Orientation relationships between different phases with different crystallographic structures can also be determined by SPED. For example, we deduced the crystallographic relationships between dispersoids (T-phase), precipitates of S-phase, and the surrounding Al matrix for T-/S-phase aggregates in an Al-Cu-Mg alloy by combining SPED, highresolution TEM and misorientation analysis in 3D axis-angle space [3]. We have also applied SPED for quantifying subgrain sizes and relative orientations of nano-sized subgrains in deformed alloys. Crystal orientations inside precipitate free zones (PFZs) in grain boundary regions in an $\mathrm{Al}-\mathrm{Mg}-\mathrm{Si}$ alloy in peak aged condition were determined after different degrees of uniaxial compression [4]. These examples demonstrate the great versatility of SPED to study and further develop aluminum alloys [8].

[1] JK Sunde et al., Materials Characterization 142 (2018), p. 458.

[2] E Thronsen et al, Effect of heavy deformation on precipitation in Al-Mg-Si-Cu, to be subm. (2019).

[3] JK Sunde et al., Acta Materialia 166 (2019) p. 587.

[4] E Christiansen et al., Materials Characterization 144 (2018), p. 522.

[5] JS Barnard, DN Johnstone, PA Midgley, Ultramicroscopy 174 (2017) p. 79.

[6] F de la Peña et al., HyperSpy - v. 1.4 (2018), doi:10.5281/zenodo.583693; (http://hyperspy.org/)

[7] https://github.com/pyxem

[8] The authors acknowledge support from The Research Council of Norway, and industrial partners through the following projects; 247783, 247598 and 237885. The SPED work was conducted using the NORTEM infrastructure (NFR 197405) at the TEM Gemini Centre, Trondheim, Norway.

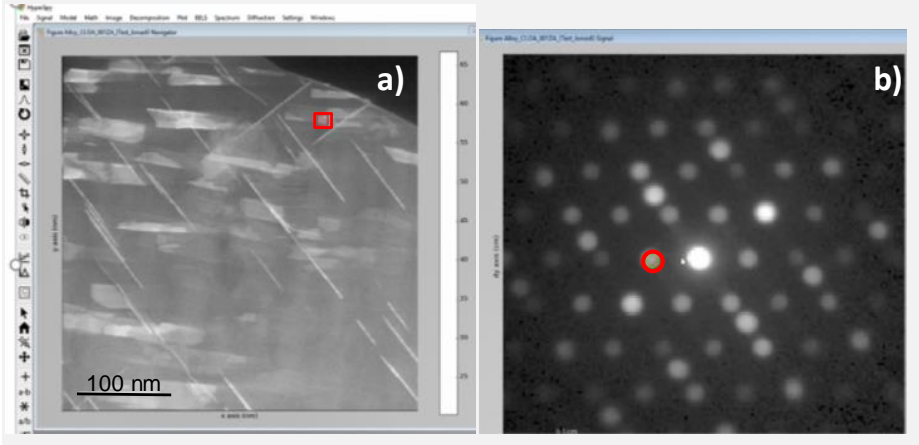

Figure 1. (a) VDF image of an Al-Cu-Li alloy in [100]Al orientation formed using the indicated virtual aperture placement in the PED pattern (b). The PED pattern corresponds to the highlighted scan pixel in (a), which coincides with a T1 precipitate phase. The SPED data is visualized using a HyperSpy-based GUI [6].

Figure 2. (a) Large area [100]Al SPED scan in an Al-Mg-Si$\mathrm{Cu}$ alloy, where $\sim 3700$ precipitates were identified and classified in three types for phase quantification.

(b) Key component patterns and corresponding lattice images of the three precipitate types.
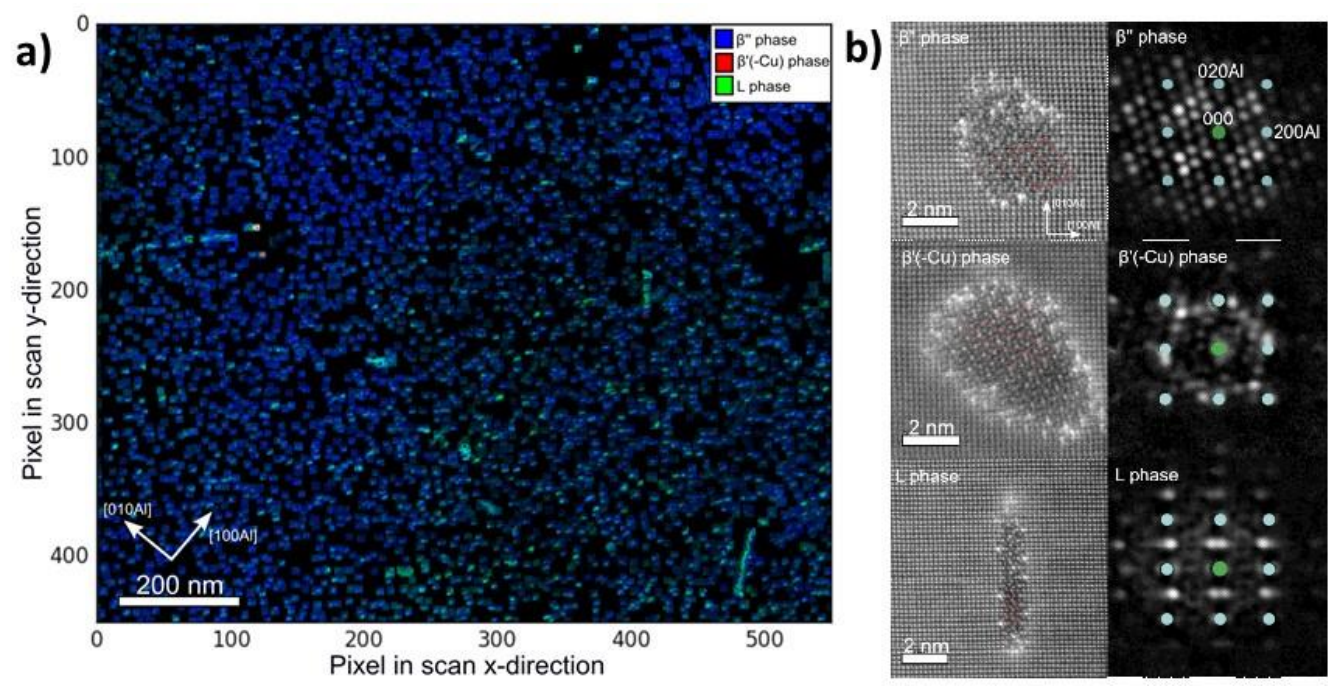